\title{
XMM-Newton Observations of Nearby Edge-On Starburst Galaxies
}

\author{
Matthias Ehle \\ XMM-Newton Science Operations Centre, European Space Agency, \\ Villafranca del Castillo, P.O. Box 50727, 28080 Madrid, Spain \\ Michael Dahlem \\ Australia Telescope National Facility, Paul Wild Observatory, Locked \\ Bag 194, Narrabri NSW 2390, Australia \\ Elena Jiménez Bailón, Maria Santos-Lleó \\ XMM-Newton Science Operations Centre, European Space Agency, \\ Villafranca del Castillo, P.O. Box 50727, 28080 Madrid, Spain \\ Andrew M. Read \\ Dept. of Physics and Astronomy, Leicester University, Leicester LE1 \\ 7RH, UK
}

\begin{abstract}
We report on the results of XMM-Newton observations of nearby starburst galaxies that form part of a multi-wavelength study of all phases of extraplanar gas in external galaxies, which is conducted in order to assess the importance of halos as repositories of a metal-enriched medium and their significance in terms of galactic chemical evolution and possible metal enrichment of the intergalactic medium (IGM). XMMNewton observations of the starburst galaxy NGC 1511 revealed e.g. the presence of a previously unknown extended hot gaseous phase of its interstellar medium (ISM), which partly extends out of the disk plane. We also present preliminary results based on XMM-Newton observations of NGC 1808, NGC 4666 and NGC 3628.
\end{abstract}

\section{Introduction}

The high sensitivity of XMM-Newton enables us to conduct observations of fainter targets than before and to obtain a much more detailed picture of those known already. Increased sensitivity leads to significant progress in investigations of low surface-brightness emitters, such as for example hot gaseous halos around actively star-forming spiral galaxies.

We have selected nearby edge-on oriented starburst galaxies to perform a multi-wavelength (radio-continuum, HI, optical and X-ray) study of all phases of extraplanar gas to investigate the dependence of galactic halo properties on the energy input rate due to star formation (SF) in the underlying galactic disk. Special care must be taken to separate halos created by a sufficiently high 

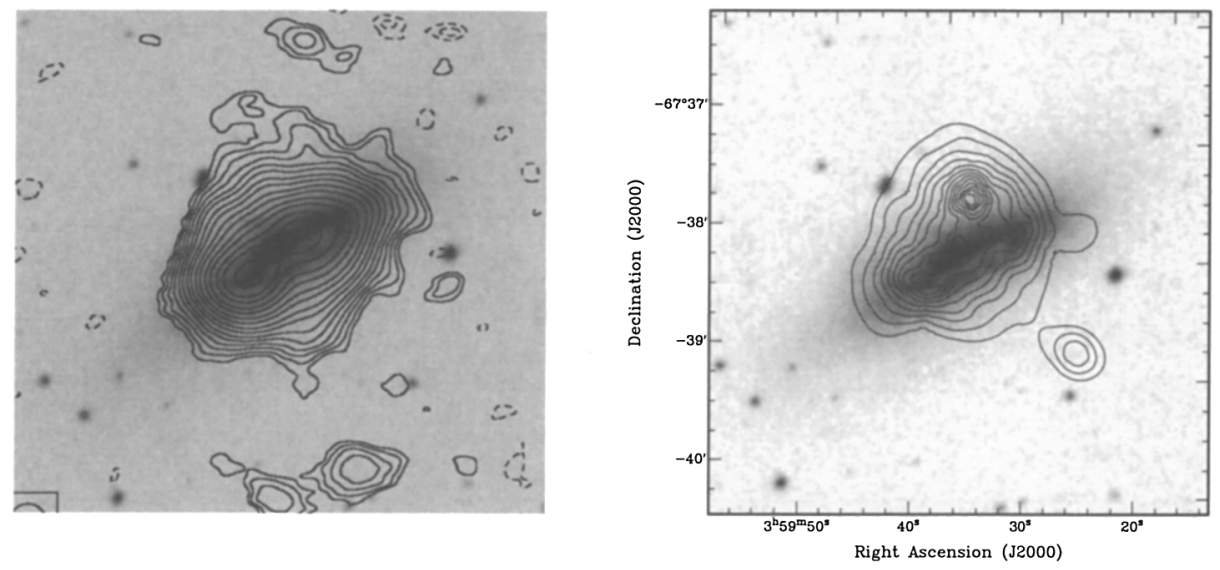

Figure 1. Radio continuum and X-ray contour maps of NGC 1511 overlaid on a DSS image. Left: ATCA $13 \mathrm{~cm}$ radio continuum contours (Dahlem et al. 2001, zoomed version of their Fig. 4 to show a similar field of view as in the right panel). Right: XMM-Newton combined pnMOS image in the $0.5-1.3 \mathrm{keV}$ energy band. The data was smoothed with an adaptive filter to enhance low surface brightness features

energy input and extra-planar emission which is caused by galactic interactions or nuclear activity. The galaxy's size, i.e. the depth of its gravitational potential, is another parameter affecting the evolution of galactic halos. One main goal of this project is the determination of the energy budget in the halos (magnetic field, thermal and radiation energy densities).

The XMM-Newton X-ray observations are used to detect previously unknown extended halo emission due to hot gas and to investigate the metallicity of the hot gas via X-ray spectroscopy.

\section{NGC 1511: The quest for hot gas in the halo}

XMM-Newton observations of NGC 1511 (Fig. 1, right panel) reveal the presence of a previously unknown extended hot gas component which is partly extending out of the disk plane. Extra-planar emission due to cosmic rays and magnetic fields is also seen in radio continuum emission (Fig. 1, left panel) and is suggestive of a common origin for the outflow of these components of the ISM. The X-ray emission distribution is asymmetric, being brightest in the eastern half of NGC 1511, where also the radio continuum emission is enhanced, indicative of a high level of star formation. The X-ray spectral analysis of the integral $0.2-12 \mathrm{keV}$ emission (excluding the strong point source about $30^{\prime \prime}$ north of the centre, which - if associated with NGC 1511 - might be an ultra-luminous $L_{X}=1.1810^{40} \mathrm{erg} \mathrm{s}^{-1} \mathrm{X}$-ray source) shows a complex emission composition: a best-fitting model was found with two thermal components and a power law contributing $12 \%(0.19 \mathrm{keV}), 11 \%(0.59 \mathrm{keV})$ and $77 \%$ (powerl) to the total flux, respectively. The corresponding total X-ray luminosity $\left(L_{X}=1.1110^{40} \mathrm{erg} \mathrm{s}^{-1}\right)$ leads to a far-infrared-to-X-ray luminosity ratio for NGC 1511 which is typical 

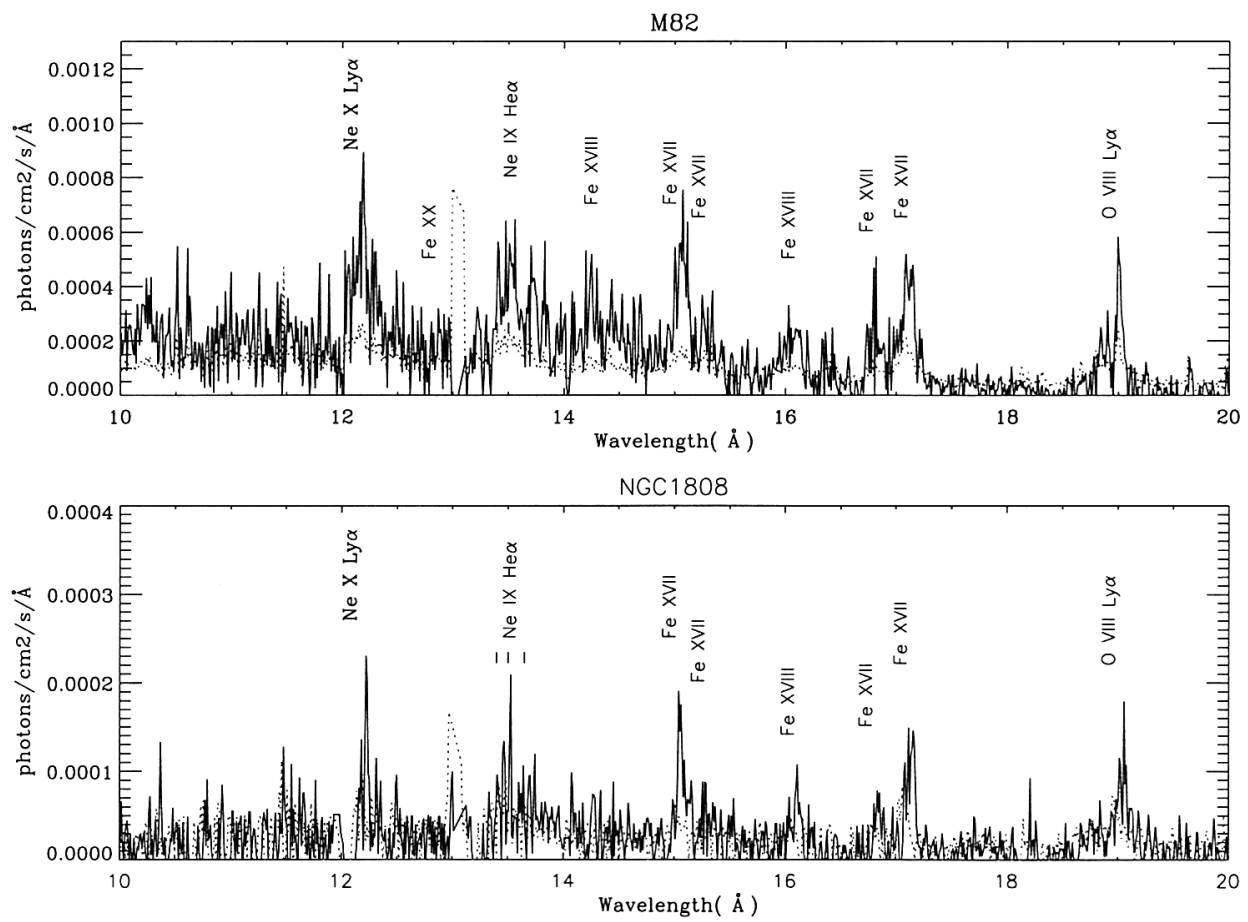

Figure 2. XMM-Newton RGS spectra of M82 (Read \& Stevens, 2002) and of NGC 1808 (see Jiménez Bailón et al. (2003, their Fig. 6)). Errors are shown as dotted line

for starburst galaxies (Heckman et al. 1990; Read \& Ponman 2001). The amount of diffuse soft $(0.2-1.3 \mathrm{keV})$ X-ray emission $\left(L_{X}=2.5510^{39} \mathrm{erg} \mathrm{s}^{-1}\right)$ is relatively low compared to other galaxies (Read \& Ponman 2001).

XMM-Newton observations with the optical monitor (OM) in the ultraviolet as well as $\mathrm{H} \alpha$ and near-infrared (2MASS) imaging suggest that NGC 1511 is heavily disturbed (most likely by its companions NGC 1511a and NGC 1511b) and hence might not be an ideal candidate to test the dependence of halo properties on the star formation in the underlying galactic disk. Still, NGC 1511 is one of a still small number of starburst galaxies in which various components of the ISM are observed, within the same volume, above their disk planes. Further details on the XMM-Newton observations of NGC 1511 have recently been published by Dahlem et al. (2003).

\section{NGC 1808: Combined starburst/AGN activity}

NGC 1808 is a galaxy well known for its high star forming activity, which might have been triggered by a recent tidal interaction with NGC 1792. Images obtained at different wavelengths suggest a high level of star formation in the central $(\approx 1 \mathrm{kpc}$ diameter) region. The nature of the nucleus remained unclear: it is classified as Seyfert 2, based on optical emission lines, but there is also 

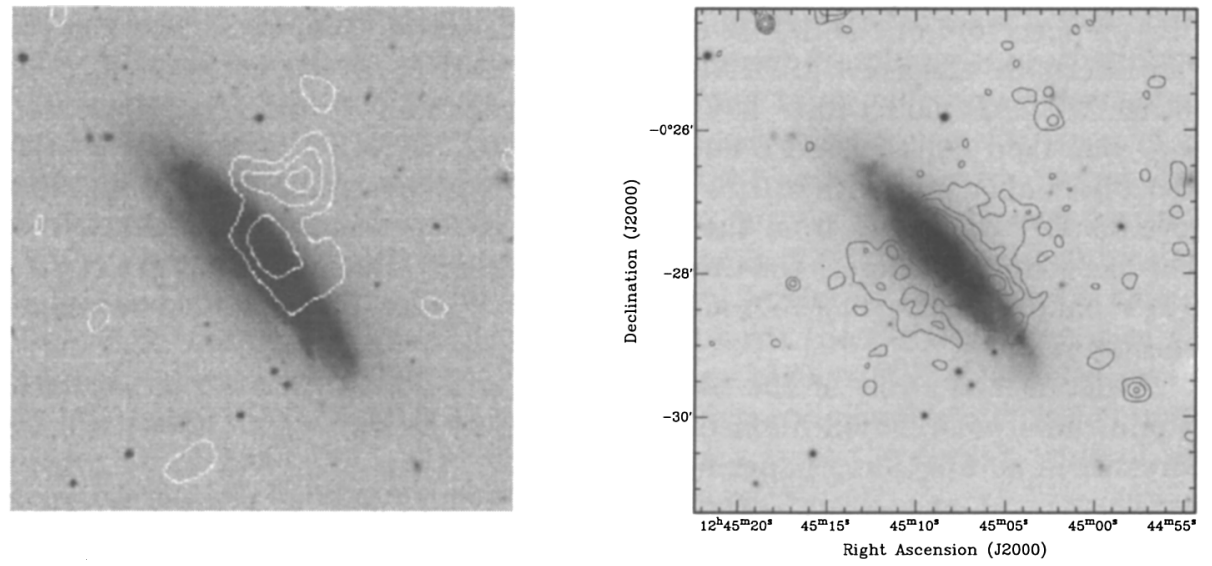

Figure 3. X-ray image of NGC 4666 overlaid on a DSS image. The displayed field of view is $7^{\prime} \times 7^{\prime}$. Left: ROSAT PSPC $0.25 \mathrm{keV}$ image by Dahlem et al. (1998, their Fig. 24). The spatial resolution is $49^{\prime \prime}$. Right: XMM-Newton combined pn-MOS image in the $0.2-0.5 \mathrm{keV}$ energy band. The data was slightly smoothed with a non-adaptive Gaussian to a spatial resolution of 10.4 "

evidence from optical polarization observations of a hidden starburst (Forbes et al. 1992). Based on ISO data, only $10 \%$ of the infra-red radiation could be interpreted as emission related to star formation (Siebenmorgen et al. 2001).

XMM-Newton data (detailed analysis in Jiménez Bailón et al. 2003 and in prep.) shed new light on the nuclear activity in NGC 1808: the EPIC-pn spectrum shows the presence of two thermal components plus an additional hard X-ray power law tail and favours the physical scenario of a co-existence of a starburst and an obscured AGN, as previously suggested by Awaki et al. (1996) based on ASCA observations. The AGN dominates the hard X-ray spectrum while emission in the soft regime (below $1 \mathrm{keV}$ ) is dominated by emission from an extended starburst, corroborating previous ASCA and ROSAT results. Both EPIC-pn and RGS data (Fig. 2, lower panel) provide reliable detections of a number of emission lines similar in wavelength and relative intensity ratios to the ones found for the prototypical starburst galaxy M82 (Fig. 2, upper panel).

\section{NGC 4666: a "Superwind" galaxy}

The high far-infrared flux ratio of $f_{60} / f_{100}=0.45$ for NGC 4666 makes this galaxy a good candidate to search for halo emission in different wavebands. In fact evidence for a starburst driven galactic superwind in NGC 4666 - based on optical emission line imagery, radio continuum maps, and soft X-ray ROSAT images (Fig. 3, left panel) - was given in Dahlem et al. (1997).

New XMM-Newton observations of NGC 4666 show for the first time that extended soft X-ray emission exists on both sides of the galactic disk originating from a huge, structured hot gas halo (Fig. 3, right panel). The complex 
EPIC-pn spectrum of the diffuse disk emission can be fitted by a combination of an internally absorbed MEKAL $(0.54 \mathrm{keV})$ and power law component plus another MEKAL model $(0.18 \mathrm{keV})$ all affected by Galactic foreground absorption. The halo emission does not need a second MEKAL component but is fitted reasonably well with a $0.22 \mathrm{keV}$ thermal plasma. Most of the total flux above $0.9 \mathrm{keV}$ originates from the power-law type emission (due to unresolved point-like sources), whereas the thermal plasma clearly dominates in the soft 0.3$0.9 \mathrm{keV}$ band (contributing $75 \%$ and $82 \%$ to the 'diffuse' disk and halo emission, respectively).

A detailed analysis of the halo emission and a possible correlation with $\mathrm{H} \alpha$ and radio polarization filaments (as started in Dahlem et al. 1997) will be addressed in an upcoming paper by Ehle et al.
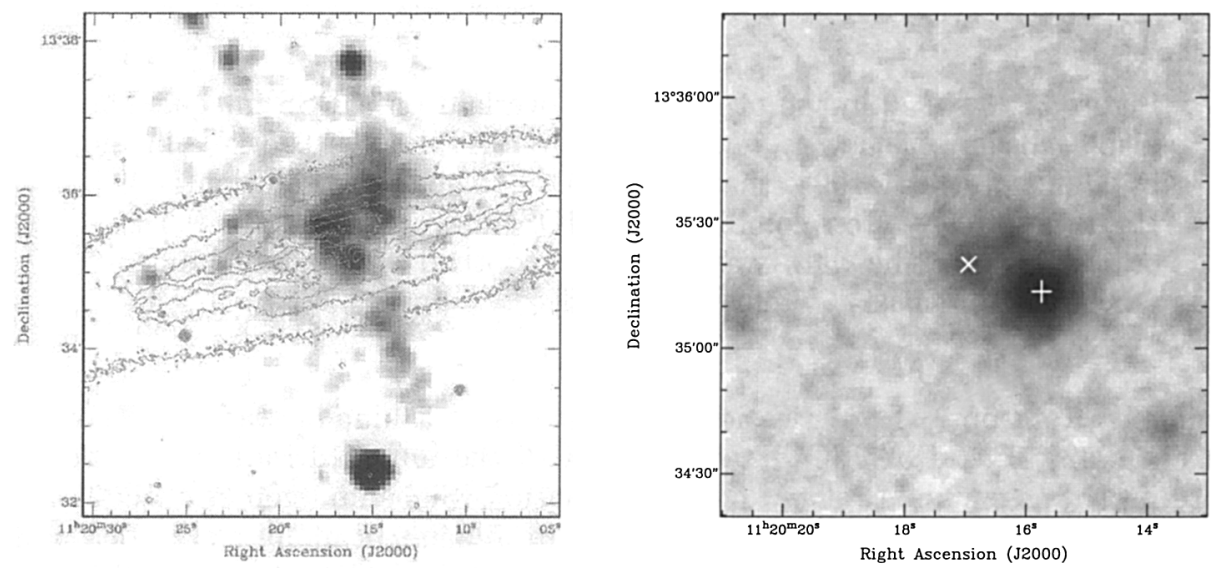

Figure 4. Left: XMM-Newton EPIC greyscale image of NGC 3628 in the $0.3-2.0 \mathrm{keV}$ band. Contours mark the distribution of the optical light and are based on a DSS image. Right: Central $2^{\prime}$ square XMM-Newton EPIC greyscale image in the $0.3-8.0 \mathrm{keV}$ band (same spatial and energy selection as the Chandra image by Strickland et al. (2001, their Fig. 1). The ' $\times$ ' marks the position of the $365 \mathrm{MHz}$ radio continuum peak, the ' + ' that of the IXO

\section{NGC 3628: outflow and a strange luminous source}

NGC 3628 is a peculiar galaxy known to be an interacting member in the Leo Triplet. Earlier X-ray observations with the Einstein (Fabbiano et al. 1990) and ROSAT satellites (Dahlem et al. 1996) showed evidence for a collimated outflow along the minor axis from a starburst nucleus in NGC 3628 and led to the detection of an extended soft X-ray halo.

Our XMM-Newton observations (Fig. 4, left panel) are able to detect the extraplanar diffuse emission with much higher significance: the EPIC image clearly separates the southern collimated spur like halo emission from nearby (most possibly background) point sources and calls the proposed link between this X-ray 
filament and QSOs (Arp et al. 2002) to question. A detailed comparison of the diffuse extraplanar X-ray emission with $\mathrm{H} \alpha$ filaments (a plume extending about $130^{\prime \prime}$ to the SW in position angle $\sim 210^{\circ}$ and faint more widespread filamentary extraplanar structures to the north - see $\mathrm{H} \alpha$ map published in Fabbiano et al. 1990) is ongoing (Ehle et al. in prep). XMM-Newton, however, is not only sensitive to the extended extraplanar emission but also to the nuclear emission region (Fig. 4, right panel). We confirm the result obtained from Chandra data (Strickland et al. 2001) which showed a very luminous point source located offset from the nucleus of NGC 3628. This source (possibly a member of the enigmatic class of intermediate-luminosity X-ray objects (IXOs)) is most probably the reason for the strong variability of the integrated nuclear emission noted already by Dahlem et al. (1995). A preliminary fit to the pn spectrum of this source is in good agreement with the Chandra results.

Acknowledgments. This work is based on observations obtained with XMM-Newton, an ESA science mission with instruments and contributions directly funded by ESA Member States and the USA (NASA).

\section{References}

Arp, H., Burbidge, E. M., Chu, Y., Flesch, E., Patat, F., \& Rupprecht, G. 2002, A\&A, 391, 833

Awaki, H., Ueno, S., Koyama, K., Tsuru, T., \& Iwasawa, K. 1996, PASJ, 48, 409

Dahlem, M., Heckman, T. M., \& Fabbiano, G. 1995, ApJ, 442, L49

Dahlem, M., Heckman, T. M., Fabbiano, G., Lehnert, M. D., \& Gilmore, D. 1996, ApJ, 461, 724

Dahlem, M., Petr, M. G., Lehnert, M. D., Heckman, T. M. \& Ehle, M. 1997, A\&A, 320, 731

Dahlem, M., Weaver, K. A. \& Heckman, T. M. 1998, ApJS, 118, 401

Dahlem, M., Lazendic, J. S., Haynes, R. F., Ehle, M., \& Lisenfeld, U. 2001, A\&A, 374, 42

Dahlem, M., Ehle, M., Jansen, F., Heckman, T. M., Weaver, K. A., \& Strickland, D. K. 2003, A\&A, 403, 547

Fabbiano, G., Heckman, T. M., \& Keel, W. C., 1990, ApJ, 355, 442

Forbes, D. A., Boisson, C., \& Ward, M. J. 1992, MNRAS259, 293

Heckman, T. M., Armus, L., Miley, G. K. 1990, ApJS, 74, 833

Jiménez Bailón, E., Santos-Lleó, M., Dahlem, M., Ehle, M., Guainazzi, M., \& Mas Hesse, J. M. 2003, astro-ph/0301109

Read, A. M., Ponman T. J., 2001, MNRAS, 328, 127

Read, A. M., Stevens, I. R., 2002, MNRAS, 335, L36

Siebenmorgen, R., Krügel, E. \& Laureijs, R. J. 2001, A\&A, 377, 735

Strickland, D. K., Colbert, E. J. M., Heckman, T. M., Weaver, K. A., Dahlem, M., \& Stevens, I. R. 2001, ApJ, 560, 707 